\title{
New Capabilities in Scanning Probe Microscopy with the Hybrid XYZ Scanner
}

\author{
T. Mueller
}

Veeco Instruments Inc., 112 Robin Hill Road, Santa Barbara, CA 93117

A new Scanning Probe Microscopy (SPM) head, the Hybrid XYZ scanner, has been introduced. The Hybrid XYZ scanner enhances our BioScope and Dimension scanning probe microscopes. Both, its technology and applications benefiting from its capabilities will be discussed.

The novel design of the Hybrid XYZ scanner interweaves elements from conventional piezo-tube and flexure based geometries (see Fig. 1), resulting in a unique combination of the advantages associated with each of these approaches. In particular, a piezo tube serves as active element providing a high response bandwidth for fast and accurate sample tracking and reduced tip wear. Flexure elements are used to transmit the motion to the SPM probe, thus affording highly orthogonal probe motion as well as the integration of opto-mechanical position sensors with high accuracy, high bandwidth and low noise. Sensors and associated closed-loop feedback are implemented for both, $\mathrm{XY}$ and $\mathrm{Z}$ motion.

The Hybrid XYZ scanner provides a unique combination of high-resolution imaging and high-resolution force spectroscopy capabilities. Images of individual biomolecules (see Fig. 2), self-assembled monolayers, and polymer blends will be presented, demonstrating open-loop and closed-loop imaging capabilities. Examples of single molecule pulling events will be shown. Single-molecule force spectroscopy benefits from the thermally-limited deflection noise in conjunction with the exceptionally low $\mathrm{Z}$ sensor noise ( $<$ 1Angstrom for a typical force ramp bandwidth) and the high accuracy and linearity over the full 8-10 micron $Z$ range. The closedloop $\mathrm{Z}$ implementation allows for true constant velocity force ramps as well as ramp scripting (see Fig. 3).

With its closed-loop on all axes and accurate sensors, the Hybrid XYZ head is particularly suitable for advanced SPM applications combining non-periodic XY and $\mathrm{Z}$ motions. Examples will be presented where high-resolution imaging is combined with point-and-shoot force ramps and with nanomanipulation.

The software package offered with the Hybrid XYZ scanner is designed to take full advantage of its capabilities. A Point-and Shoot interface optimizes the combination of force ramps with imaging. All realtime control and offline analysis features previously available with the PicoForce scanning probe microscope are incorporated, including a strip chart function, ramp scripting, and built-in thermal tuning for the calibration of cantilever spring constants. In addition, our Nanoman interface is available for taking full advantage of closed-loop on all axes during nanolithography and nanomanipulation experiments. 


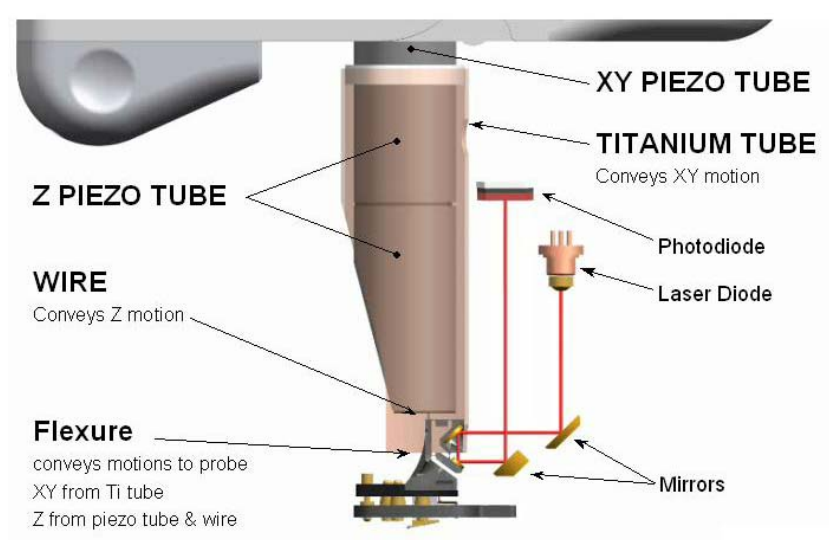

Fig.1. Inside view of the new Hybrid XYZ scanner, highlighting the role of piezo tube and flexure elements and the implementation of the $\mathrm{Z}$ sensor.

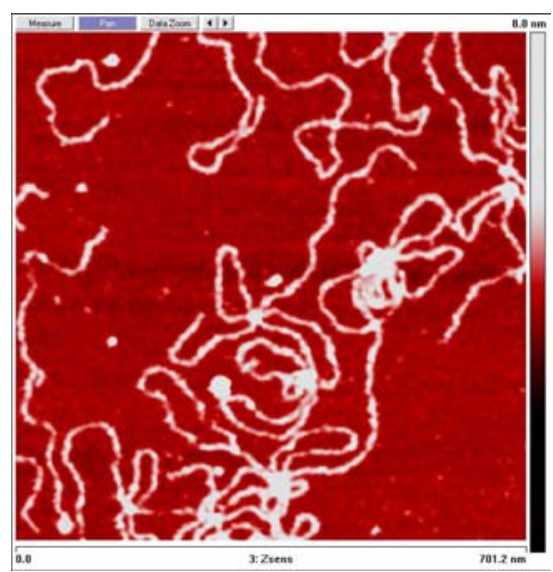

Fig. 2. High-resolution imaging of DNA in fluid. The image was acquired with the $\mathrm{XY}$ closed-loop turned on and Z-sensor data is displayed.

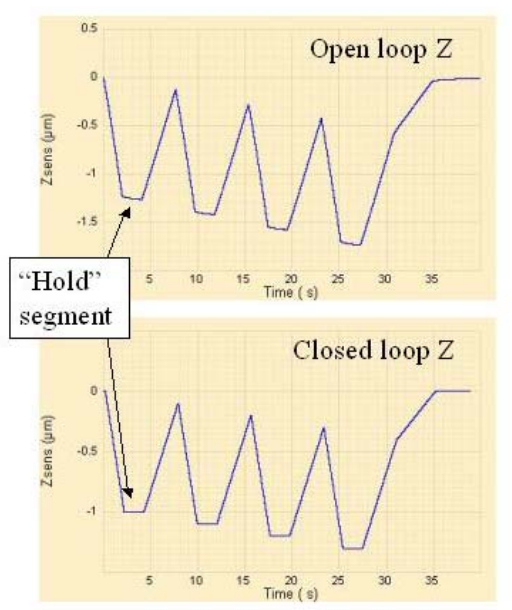

Fig. 3. Ramp scripting in open-loop and closed-loop Z mode. Shown is the Zposition (measured with the Z-sensor) as a function of time. The importance of closed-loop $\mathrm{Z}$ control for removing the effects of piezo creep is evident, particularly during the hold segments. 